\title{
Local Structure of Metastable Cobalt Complexes Studied by Resonant X-ray Diffraction
}

\author{
Marcos A. Ribeiro ${ }^{I}$, Thiago M. Francisco ${ }^{1}$, Frederico A. Lima ${ }^{2}$ and Carlos B. Pinheiro ${ }^{1}$ \\ 1- Department of Physics, Universidade Federal de Minas Gerais, Belo Horizonte - MG, Brazil. \\ 2- European XFEL GmbH, Schenefeld, Germany \\ Presenting author email: ramribeiro@gmail.com
}

Keywords: Valence tautomerism, dioxolene cobalt complex, anomalous dispersion.

Octahedral cobalt complexes with $o$-dioxolene (o-diox) ligands can present tautomeric valence equilibrium between $h s-\mathrm{Co}^{2+}$ and $l s-\mathrm{Co}^{3+}$ states in solution and solid state. In general, chemical factors like the nature of the redox-active and ancillary ligands, the presence of counter ions and solvent molecules in the lattice, as well as intermolecular interactions, influence the very existence of the valence tautomeric (VT) interconversion and are still subject of intense study[1]. External physical factors or stimuli such as temperature and pressure changes and light irradiation can modulate the tautomeric states, making this class of compounds ideal for applications in switchable molecular devices. In the solution phase, the $[\mathrm{Co}(o-$ diox $\left.)_{2}(\text { py })_{2}\right]$ complex $\left(o\right.$-diox $=3,5$-di-t-butylsemiquinonate $\left(\mathrm{SQ}^{\circ}\right)$ radical and/or 3,5-di-t-butyl-catecholate $\left(\mathrm{Cat}^{2-}\right)$; py $=$ pyridine) show no VT interconversion. In the crystalline phase, thanks to a cocrystalized pyridine molecule, $\left[\mathrm{Co}(o \text {-diox })_{2}(\mathrm{py})_{2}\right] \cdot \mathrm{py}(\boldsymbol{I})$ shows a thermo-activated tautomeric interconversion for only one of the two independent molecules in the asymmetric unit[2] (Figure 1). This work aims to quantitatively elucidate the $l s-\mathrm{Co}^{3+}$ and $h s-\mathrm{Co}^{2+}$ mole fraction changing with temperature and for that we performed a detailed study of the structure of (1) using resonant X-ray diffraction techniques in the temperature range between $300 \mathrm{~K}$ and $100 \mathrm{~K}$ [3]. The previous works on 1 [2] define the oxidation and spin state of the Co ions in the structure based on average properties such as the Co-Ligand interatomic distances and sample magnetic susceptibility. Despite correct, these approaches lack information of the local electronic structure of the cobalt ion during the interconversion.

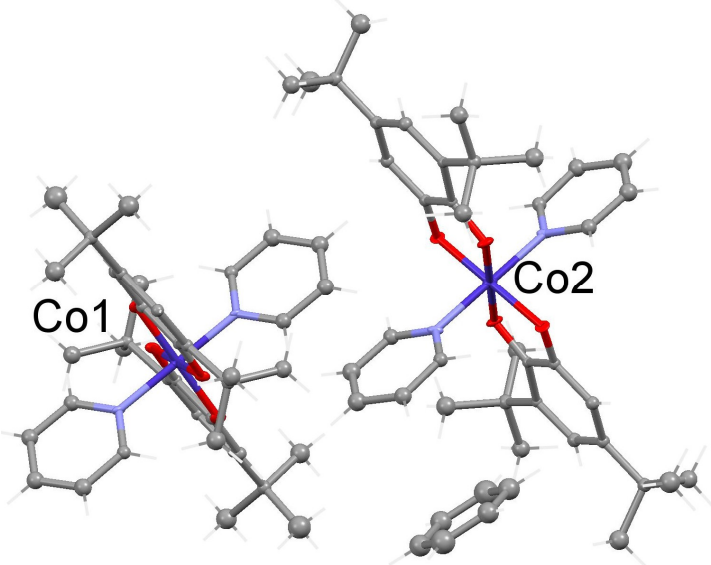

Figure 1: $\left[\mathrm{Co}(o-\operatorname{diox})_{2}(\mathrm{py})_{2}\right] \cdot$ py asymmetric unit

[1] Tezgerevska, T. et al.(2014). Coordination Chemistry Reviews, 268, 23-40.

[2] Mulyana, $Y$. et al. (2009). Dalton Trans.,39, 4757-4767

[3] Einsle, O. et al. (2007). J. Am. Chem. Soc., 129, 2210-2211. 